$>$ Nous proposons, dans cet article, des exemples d'applications médicales de la biologie des systèmes, en rappelant d'abord la problématique temporelle de l'observation médicale et le cadre général des systèmes dynamiques, puis en montrant quelles techniques provenant de la théorie des systèmes complexes peuvent s'appliquer dans divers domaines de la médecine : l'immunologie, la génétique médicale, la morphogenèse, la biochimie médicale, l'épidémiologie, la télémédecine et la pluri-expertise. Nous terminons en posant le problème du dossier patient «clinomique », réunissant le dossier clinique et les données «omiques».<

\section{Biologie des systèmes et applications médicales}

Jacques Demongeot

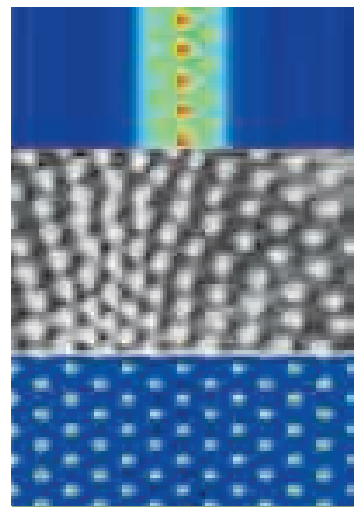

TIMC-IMAG UMR UJF/CNRS 5525, Université J. Fourier de Grenoble, Faculté de médecine, 38700 La Tronche, France. jacques.demongeot@imag.fr
L'évolution actuelle de la médecine moderne vers une médecine des «4P », c'est-à-dire personnalisée, préventive, plurielle (pluri-experte) et prédictive, passe par la mise en place d'un référentiel commun, à la fois référentiel temporel, mettant en correspondance le chronos des instruments d'observation, le kairos du temps biologique du malade et l'aion de la réflexion médicale (aion ou dasein, temps immanent, substrat de la psyché d'Aristote), mais également référentiel systémique, décrivant les différents systèmes impliqués dans une pathologie, dans la dynamique de leurs interactions, et enfin référentiel cognitif, inscrivant les différents savoirs médicaux (savoir encyclopédique d'une université médicale virtuelle, savoir statistique de bases de données patients et savoir expert de spécialistes) dans un même gisement, distribué et partagé, de connaissances en santé. Ce défi (harmoniser temps, systèmes et savoirs), la médecine moderne doit le relever, et munie de ce référentiel commun, elle pourra satisfaisaire à la fois les exigences d'atteinte du bien-être individuel de Panacea et du bon état sanitaire collectif d'Hygeia, les deux déesses grecques de la santé. Nous donnerons, dans la suite, des exemples qui s'inscrivent dans le paradigme des systèmes dynamiques complexes, cumulant les difficultés liées aux changements d'échelle et de temps, mais permettant d'interpréter, dans une méthodologie dite «biologie des systèmes ", les comportements émergents de la morphogenèse physiologique dont la connaissance des mécanismes est indispensable à la compréhension de la dysgenèse pathologique.

\section{La temporalité de l’observation médicale}

La temporalité de l'observation médicale est essentiellement liée à la perception différentielle du temps par le patient et par le thérapeute. Le temps est en effet figé dans la médecine classique hippocratique qui observe le patient à travers le filtre du chronos (temps régulier d'une chronique). Hippocrate de Chios (460-370 av. J.-C.) fonda une école médicale à Epidaure (Figure l) et remarqua qu'une maladie ne se déclarait pas brusquement, mais passait par différentes phases, aboutissant à une période dangereuse : la «crise ». II nota que, dans l'évolution des maladies, surtout celles associées à une fièvre, les quatrième, onzième, quatorzième et vingtième jours étaient des jours particulièrement à risque de «crise ». Hérophile d'Alexandrie (331-250 av. J.-C.), éduqué par Praxagoras à Cos, a introduit le premier une notion du temps non chronologique, celle du kairos (temps opportun où un patient délivre un symptôme et consulte, et où un médecin l'observe), lié aux perceptions qu'a le malade de sa propre maladie et à la succession des sympômes qui en découle. II travailla à Alexandrie sous Ptolémée I Soter (323-283) et Ptolémée II Philadelphe (283-246) et fut le premier à prendre conscience de la pulsation artérielle 


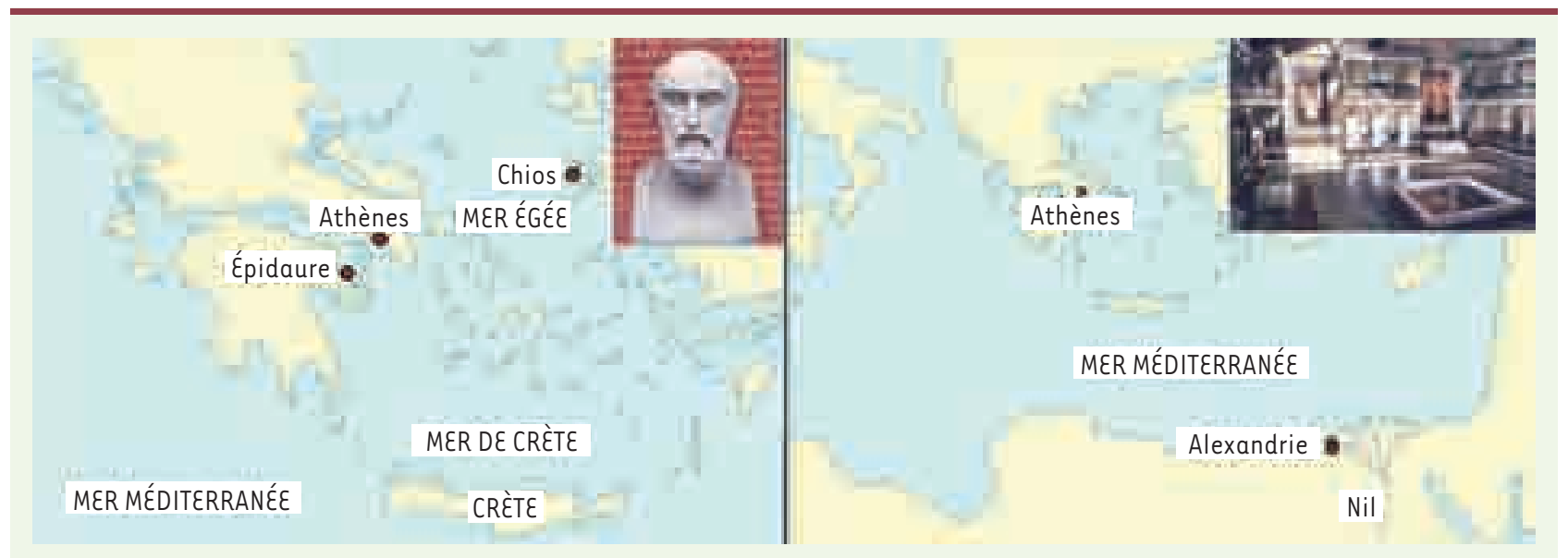

Figure 1. La médecine grecque, née à Epidaure dans sa dimension «chronologique » et à Alexandrie dans sa dimension «kairotique 》 (buste d'Hippocrate en haut à gauche et reconstitution de la bibliothèque d'Alexandrie en haut à droite).

au poignet et à en fournir une explication «mélodique», soumise à des rythmes dépendant de l'âge, du sexe et du tempérament de chaque individu : il mesurait les différentes pulsations (au repos, à l'effort, en crise fébrile, etc.) en utilisant un instrument spécial ressemblant à une petite clepsydre. Hérophile est le père de la médecine dynamique qui met les instants d'apparition des sympômes et de délivrance médicamenteuse au centre d'un schéma d'observation à des instants non équidistants que l'on appelle, de manière paradoxale, la chronomédecine. Aujourd'hui, on mesure une très grande variété de rythmes, en particulier ceux dont la période couvre la durée du nycthémère (alternance jour/nuit), c'est-àdire 24 heures, comme la température et le rythme cardiaque (Figure 2). Le fait sanitaire saillant, à un kairos donné, est une singularité qui permet de mieux comprendre les mécanismes des rythmes biologiques et donc de corriger leurs pathologies. II s'agit, au niveau individuel, de l'apparition d'un syndrome (c'est-à-dire d'un ensemble cohérent de symptômes

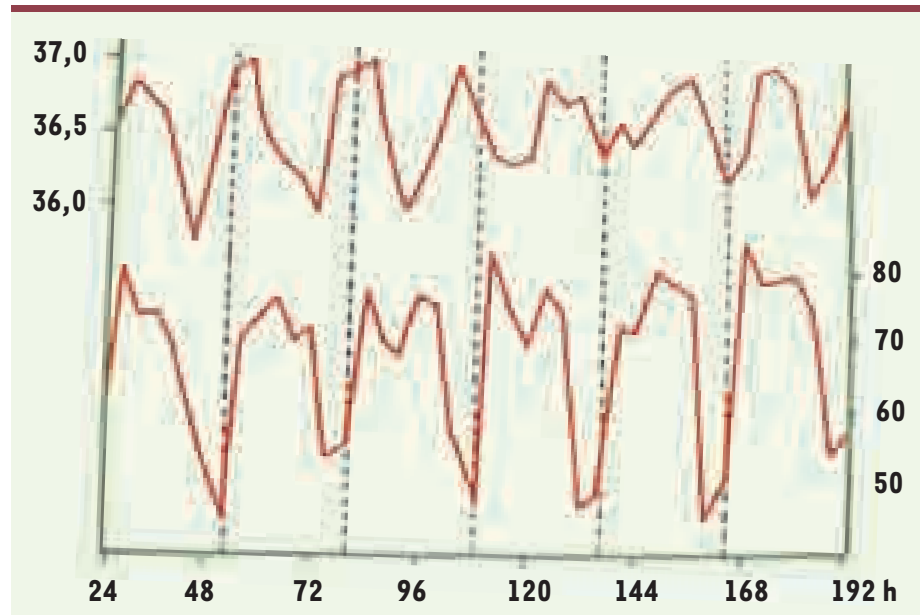

Figure 2. Oscillations nycthémérales de la température orale en degrés Celsius (en haut) et du nombre de battements cardiaques par seconde (en bas) chez l'homme. évoquant en général une maladie, que la démarche diagnostique a pour but d'orienter, puis d'affirmer) et, au niveau collectif, de l'occurrence d'un fait de santé publique, par exemple une épidémie. Le fait sanitaire saillant a une origine (étiologie), s'inscrit dans une histoire passée (anamnèse) et dans un terrain (par exemple familial). II consiste en une succession de kairos (par exemple les instants des pics de fièvre du V grippal ou de l'entrée dans la phase contagieuse, Figure 3) et s'achève par une guérison, à la suite d'un traitement délivré à intervalles fixes (thérapie traditionnelle hippocratique) ou d'un traitement lié à l'apparition des symptômes («kairothérapie», appelée paradoxalement chronothérapie), ou par un passage à la chronicité, avec pour seul succès la disparition des signes les plus invalidants (traitement symptomatique). L'ensemble des données d'anamnèse (individuelle et collective, en général cliniques) et de terrain (familial, en général génétiques) constitue la prégnance sanitaire qui est consignée dans le carnet de santé personnel (observation d'un individu) ou dans des bases de données sanitaires collectives (observation d'une population).

Plus généralement, les événements kairotiques purs sont par exemple la survenue, au stade adulte, d'un zona, rappel d'une varicelle survenue au stade de développement, ce dernier incident étant mixte car il apparaît en général à un instant du chronos social: celui de la scolarisation. Un véritable dossier de santé individuel (dont nous n'avons que l'ébauche dans le carnet de santé de l'enfant) devrait comporter la possibilité d'inscrire les kairos sanitaires dans la chronologie sociale d'un sujet et dans sa prégnance génétique (Figure 4).

Le temps psychologique du patient s'inscrit dans l'aion aristotélicien et donne lieu à des représentations du 


\begin{tabular}{|c|}
\hline Incubation \\
\hline
\end{tabular}

Figure 3. La succession des phases (en haut) et des états (en bas) d'une maladie infectieuse. nombres (ou heures) de 1 à 12 chez un héminégligent droit. La restitution de l'anamnèse ou la description de la crise prend évidemment pour support, chez le patient, ces images mentales dont il faut évaluer la distorsion, en cas de désorientation temporo-spatiale, par exemple chez le patient confus, victime d'un accident vasculaire cérébral ou d'une maladie dégénérative chronique.

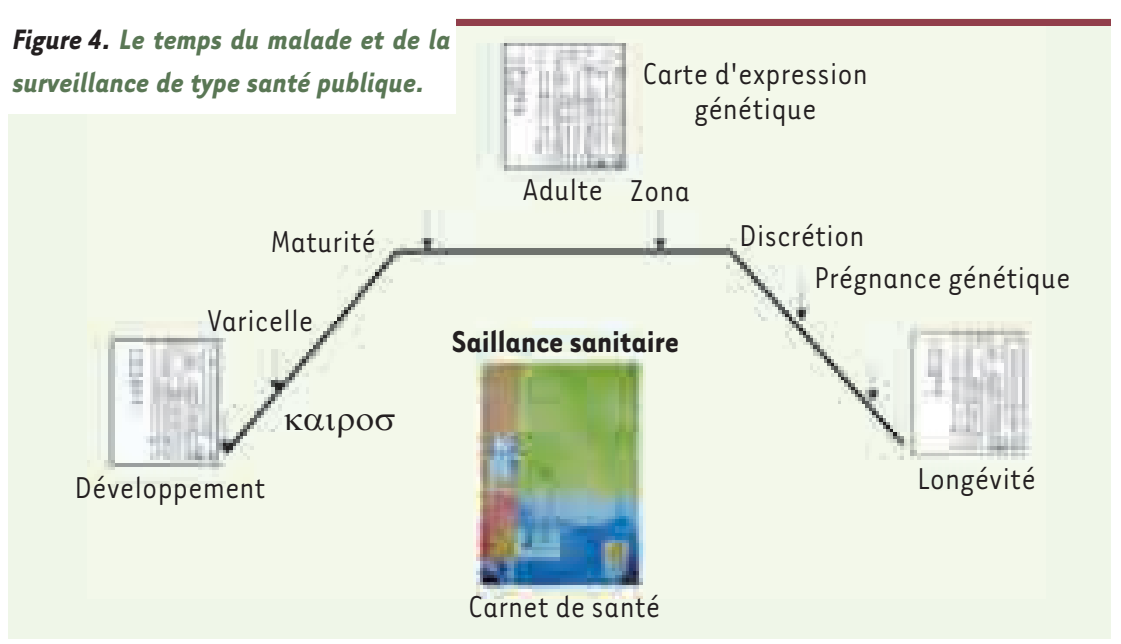

\section{Les systèmes dynamiques et les systèmes complexes}

L'un des outils mathématiques les plus utilisés pour étudier les systèmes complexes de régulation en médecine, à quelque niveau que ce soit (physiologique, métabolique, génétique, etc.) est la théorie des systèmes dynamiques [5] et son complément moderne: la théorie des systèmes complexes [6]. Ces théories expliquent comment, à partir d'interactions connues entre les éléments d'un système évoluant au cours du temps (organes, protéines, gènes, etc.), apparaissent des états dits «attracteurs », uniques états visibles phé- temps par rapport auxquelles le patient s'oriente; ces représentations et les images mentales dont on demande la recopie sur papier au patient sont normales ou pathologiques: la Figure 5 (en haut à gauche) donne ainsi une représentation des nombres (ou des minutes) de 1 à 100 chez une personne normale, étudiée dès 1879 par F. Galton [1], puis, depuis cette date, par de nombreux psychologues $[2,3]$; elle montre, à droite, la représentation de l'année du peintre Paul Klee [4], puis, en bas à gauche, une représentation pathologique des nombres (ou des heures) de 1 à 100 , chez un patient souffrant d'une héminégligence gauche et enfin, en médaillon, les

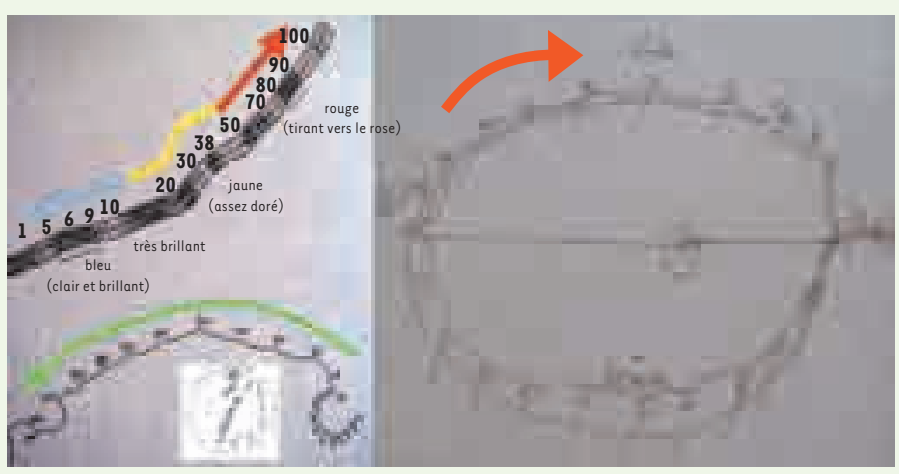

Figure 5. Le temps du malade et la représentation subjective des kairos du bienêtre et de la maladie. image mentale du temps chronologique normal (en haut à gauche), du temps chez un héminégligent gauche et droit (en bas à gauche et médaillon) et représentation de l'année de P. Klee (à droite). notypiquement, quelles que soient les conditions initiales du système, à condition que celles-ci soient situées dans un ensemble de perturbations admissibles de l'attracteur, appelé son bassin de stabilité. Ainsi, si le système dynamique est un réseau de régulation génétique, les états des gènes peuvent prendre deux valeurs: 1 si le gène s'exprime et - 1 s'il est silencieux. À partir d'une configuration initiale, on cherche alors à obtenir son état attracteur (les gènes devant changer d'état pour l'atteindre étant marqués en rouge, Figure 6), en imposant au système de respecter une règle de majorité : on donne à un gène l'état de la majorité de ses voisins, chacun de ceux-ci essayant de lui conférer son état s'il l'active (noté + sur le graphe d'interactions génétiques, Figure 6) ou l'opposé de son état s'il l'inhibe (noté -). En cas d'égalité, on choisit l'état 1.

$\varepsilon n$ étudiant systématiquement tous les attracteurs possibles, par exemple dans un réseau de régulation morphogénétique [7], on identifie ceux qui correspondront à la phénoménologie observée, par exemple l'apparition des phanères: plumes chez les oiseaux, et poils et vibrisses chez les mammifères (voir plus loin, Figure 16). Ces attracteurs doivent être très robustes [8] pour résister aux perturbations de l'environnement sur l'architecture des interactions (à laquelle les attracteurs sont très sensibles), par exemple par intervention de microARN, souvent issus d'agents infectieux intervenant au cours de l'évolution des espèces concernées $[9,10]$. Cette robustesse devient vitale lorsque la morphogenèse concerne un organe dont la fonction est critique pour la survie, par exemple la maintenance mitochondriale de l'intégrité respiratoire cellulaire [11] ou le contrôle de la morphogenèse par des facteurs de transcription [12]. 
Lorsque le nombre $\mathrm{n}$ de gènes augmente se pose le problème de la dépendance des attracteurs (nombre et nature) vis-à-vis de la frontière du graphe d'interaction [26-28]. Les gènes non inclus dans les composantes connexes du

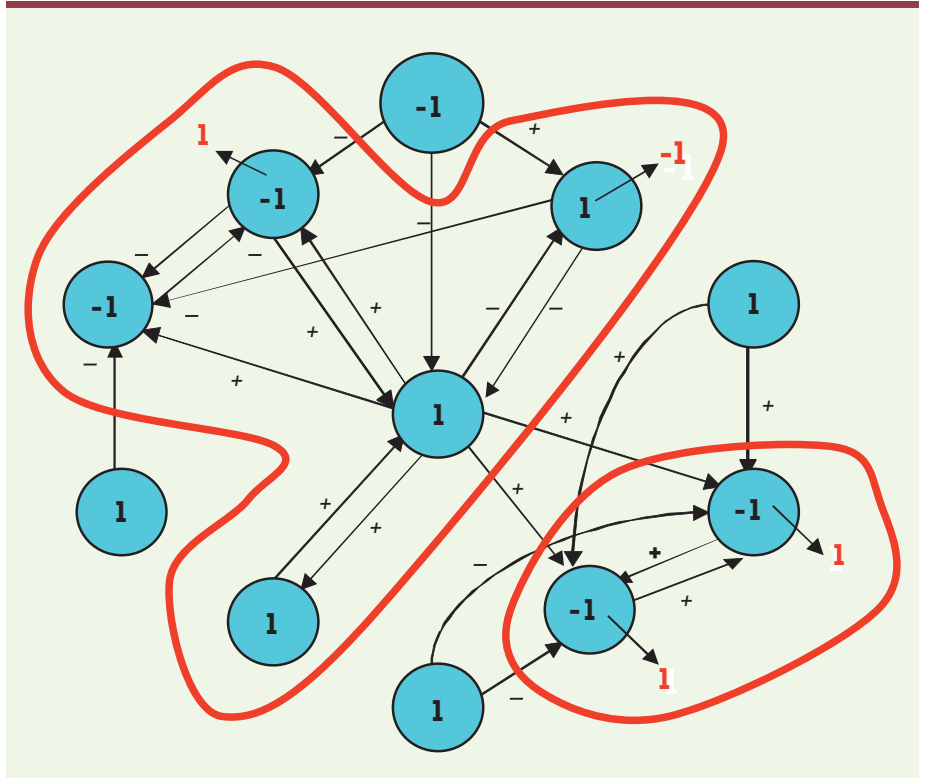

Figure 6. Le graphe d'interactions génétiques du contrôle de la floraison d'Arabidopsis thaliana. Les deux composantes connexes du graphe sont entourées de rouge. Une condition initiale d'états est notée en noir sur les sommets du réseau et les états de son attracteur sont notés en rouge (lorsqu'ils diffèrent de ceux de la condition initiale).

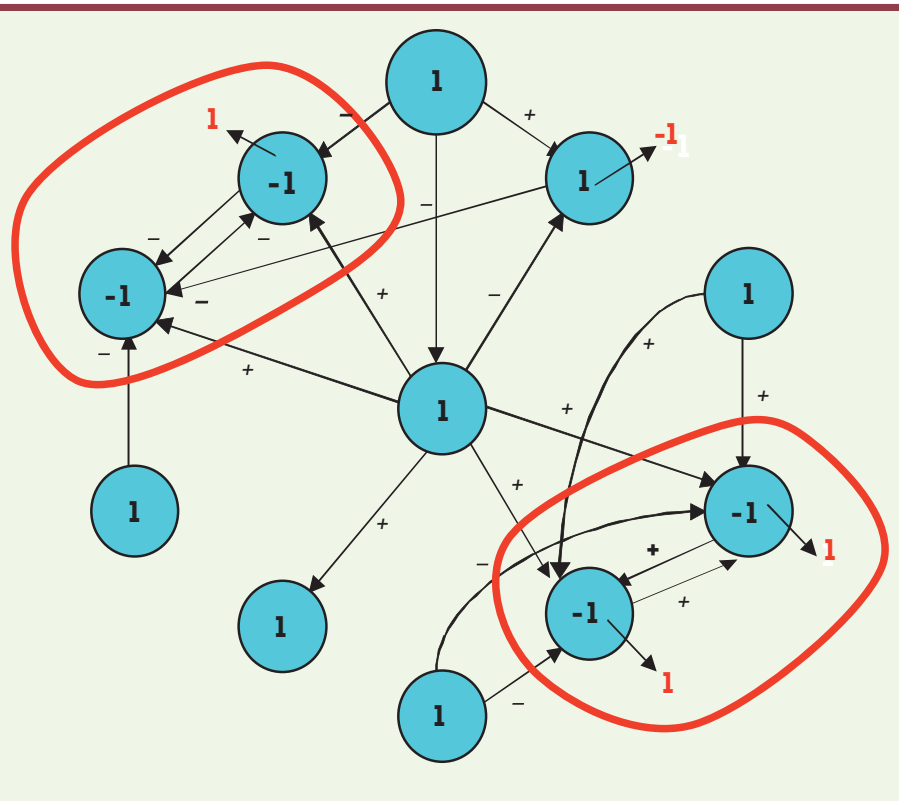

Figure 7. Graphe de la Figure 6 simplifié, ne comportant plus que 2 composantes fortement connexes simples (boucles positives symétriques, en rouge), et gardant les mêmes attracteurs. graphe d'interactions de la Figure 6 sont des «jardins d'Eden » (on en part, mais on n'y revient jamais) et constituent la frontière du graphe. II existe des gènes frontières critiques, ce sont ceux qui, lorsque l'on change définitivement leur valeur (en la mettant à zéro après une invalidation génique par exemple, ce qui provoque la disparition des interactions qu'ils exercent sur les autres gènes), provoquent un changement profond du comportement $\mathrm{du}$ réseau (changement des bassins de stabilité) ou un changement du nombre ou de la nature de ses attracteurs (qui peuvent passer par exemple d'une configuration fixe à un cycle de configurations). Lorsque n croît, il est nécessaire de simplifier les graphes, en ne gardant que les interactions provenant des gènes de la frontière (jardins d' $\varepsilon$ den) et celles du cœur du réseau, souvent organisés en motifs simples interconnectés, cela sous la condition expresse de conserver les mêmes attracteurs et bassins. Par exemple, le graphe de la Figure 7 a la même dynamique que celui de la Figure 6, mais son cœur ne comporte que des motifs de type boucle positive symétrique. Un résultat ancien [19] prouve que seuls sont alors observables, en plus des configurations fixes, les cycles d'ordre 2, ce qui est effectivement le cas dans les simulations de la dynamique des Figures 6 et 7, dans tous les modes d'itération (séquentielle, correspondant à une mise à jour successive de tous les gènes du réseau, et parallèle, correspondant à une mise à jour synchrone), les cycles n'apparaissant que dans le mode parallèle, qui est exclu du fait du schéma admis chez Arabidopsis d'une coexpression séquentielle des gènes par blocs (Figure 8) [29, 30].

\section{Applications de la biologie des systèmes en immunologie médicale}

Un exemple d'application est donné par la base IMGT (Immunogenetics database) qui recense tous les cas de réarrangements du génome à l'origine du répertoire des cellules immunitaires; ce génome, localisé sur le chromosome 14 chez l'homme, code pour de nombreuses protéines du répertoire immunologique dont la présence conditionne la qualité de la réponse aux maladies infectieuses. Prévoir cette réponse consiste à comparer les anticorps circulants d'un patient à ceux qui sont attendus dans le cas d'un système immunologique normal. Toute perte d'intégrité de ce dernier sera détectée par comparaison avec un matériel immun normal, décrit, dans sa variabilité statistique, par les mécanismes et les résultats des réarrangements les plus probables du chromosome 14. Cette description est accessible en interrogeant la base de référence mondiale IMGT (Figure 9) [31, 32] et permet la construction de systèmes dynamiques complexes gouvernant par exemple l'activation des lymphocytes $T$ [33]. 


\section{Représentation mathématique d'un système dynamique complexe}

Un système dynamique complexe évolue en général dans un contexte multi-échelle : par exemple, une partie de sa fonction de transition $F$, qui permet de passer de l'état $x(t)$ du système à l'état suivant $x(t$ $+d t$ ) ou $x(t+1)$, selon que le système est continu ou discret, peut évoluer avec un temps $t$ lent et l'autre partie avec un temps rapide $t / \varepsilon$, où $\varepsilon$ est petit; suivant le cas (continu ou discret), nous aurons donc, si $x(t)=\left(x_{1}(t), x_{2}(t)\right)$ est le vecteur d'état, constitué des deux sous-vecteurs $x_{1}(t)$ lent et $x_{2}(t)$ rapide: $d x / d t=F(x(t)) \Leftrightarrow$ $\mathrm{dx}_{1} / d t=\mathrm{F}_{1}(\mathrm{x}(\mathrm{t})), \mathrm{dx}_{2} / \mathrm{dt}=\mathrm{F}_{2}(\mathrm{x}(\mathrm{t})) / \varepsilon($ resp. $\mathrm{x}(\mathrm{t}+\mathrm{l})-\mathrm{x}(\mathrm{t})=\mathrm{F}(\mathrm{x}(\mathrm{t})) \Leftrightarrow$ $\left.x_{1}(t+1)-x_{1}(t)=F_{1}(x(t)), x_{2}(t+1)-x_{2}(t)=F_{2}(x(t)) / \varepsilon\right)$, en continu (resp. discret).

Le système dynamique complexe présente alors une dynamique rapide pour sa variable $x_{2}$ et une dynamique lente pour sa variable $x_{1}$, qui a lieu sur l'ensemble $F_{2}(x(t))=0$, appelé variété centrale $d u$ système. Au voisinage des états stationnaires $x^{\star}$ du système (qui vérifient $F\left(x^{\star}\right)=0$ ), le système continu peut localement se développer en série :

$\mathrm{dx} / \mathrm{dt}=\mathrm{F}\left(\mathrm{x}^{\star}\right)+J\left(\mathrm{x}^{\star}\right)\left(\mathrm{x}(\mathrm{t})-\mathrm{x}^{\star}\right)+0\left(|| H\left(\mathrm{x}^{\star}\right)|| .|| x(\mathrm{t})-\left.\mathrm{x}^{\star}\right|^{2}\right)$, où $J$ (resp. H) représente la matrice jacobienne (resp. le tenseur hessien) du système, c'est-à-dire la matrice de terme général: $J_{i j}=\partial F_{i} / \partial x_{j}\left(x^{\star}\right)\left(\right.$ resp. $\left.H_{i j k}=\partial^{2} F_{i} / \partial x_{j} \partial x_{k}\left(x^{\star}\right)\right)$ et ||$H||$ est la norme de $H, c^{\prime}$ est-à-dire le maximum de la valeur absolue de ses coefficients.

On appelle graphe d'interactions du système le graphe dont la matrice incidente est $\mathrm{J}$ : ce graphe a une flèche munie d'un signe + (resp. -) entre ses sommets $i$ et $j$, si $j_{i j}>0($ resp. $<0)$ et n'a pas de flèche, si $j_{i j}=0$. Dans l'exemple de la Figure 6, le graphe d'interactions correspond à la régulation de la floraison chez Arabidopsis thaliana et il sert souvent d'exemple pour les études de la dynamique d'un système de régulation génique [8]. Ce graphe possède deux composantes fortement connexes (entourées de rouge sur la Figure 7), c'est-à-dire deux sousensembles de gènes dont tout couple de sommets peut être relié dans les deux sens par un chemin orienté (succession de flèches orientées). Les composantes fortement connexes importantes sont celles qui contiennent des circuits positifs (c'est-à-dire des chemins orientés bouclés, partant d'un gène et revenant sur lui-même, et comportant un nombre pair d'interactions négatives); en effet, leur décompte permet de majorer le nombre des attracteurs du réseau. II est très important de décompter ces attracteurs, car, depuis la remarque séminale de M. Delbrück en 1949, on peut assimiler leur nombre à celui des types cellulaires différenciés contrôlés par le réseau de régulation $[13,14]$. De récents résultats permettent d'envisager une connaissance précise des rapports entre le nombre d'attracteurs et celui des fonctions codées dans un réseau de régulation. Soit $\mathrm{C}$ le nombre de ses composantes fortement connexes comportant un circuit positif, A le nombre de ses attracteurs, $K=I / n$ sa connectivité, c'est-à-dire le rapport entre les nombres I d'interactions et $n$ de gènes, et $P$ le nombre de ses circuits positifs, en ne comptant qu'une fois ceux qui partagent un gène. Nous avons alors les conjectures: $2^{P} \geq A \geq 2^{C}$ et $A \geq 0\left(n^{1 / 2}\right)$, valables dans les graphes de type Hopfield, dont les fonctions de transition sont du type majorité pondérée [15-22] et dans les graphes de type Kauffman, dont les fonctions de transition sont toutes les fonctions booléennes possibles [6, 23-25]. Dans les réseaux constitués de couches successives de gènes se co-exprimant, on peut montrer que la borne supérieure peut être remplacée par $2^{v}$, où $V$ est le nombre de gènes de la première couche [22].

\section{Applications de la biologie des systèmes en génétique médicale}

Si la prégnance génétique est constituée par l'ensemble des gènes normaux d'une population, la saillance d'un ou plusieurs gènes pathologiques (par suite de mutation, translocation, inversion, insertion et/ou délétion de portions de chromosomes, entre deux générations ou durant une maladie chronique comme le cancer), permet d'expliquer une phénoménologie anormale. Un exemple de cas de saillance est donné par la constatation clinique d'une spina bifida (Figure 10), suivie par la recherche du pedigree du patient obtenu lors de l'interrogatoire de l'anamnèse, cherchant à recenser les parents présentant la même pathologie, puis par l'identification des gènes concernés dans la famille, et enfin par la confrontation avec des familles de référence présentant la même phénoménologie, par exemple en utilisant la base de données HCForum ${ }^{\circledR}$ [34].

Les sources de saillance sont multiples, allant d'une expression génétique témoin d'une dysrégulation de type sur-expression (up-regulation) ou sous-expression (down-regulation) se traduisant respectivement par un pic ou une lacune dans le spectre protéique, jusqu'à la manifestation de la maturation (physiologique ou non) d'une fonction (puberté), en passant par l'atteinte de limites physiologiques (comme la limite supérieure du nombre de mitoses permises dans un tissu, dite limite de Hayflick).

\section{Applications de la biologie des systèmes en morphogenèse}

\section{La séquence euplasie/hyperplasie/ métaplasie/dysplasie/néoplasie}

La saillance peut naître d'un processus de cancérisation dans une évolution allant du normal au pathologique, déroulant la séquence euplasie/hyperplasie/métaplasie/dysplasie/néoplasie. L'euplasie est un terme utilisé pour décrire l'état d'activité de base normal d'une cellule saine. L'hyperplasie est une augmentation du nombre de cellules dans un tissu cellulaire (collection de cellules). La métaplasie est la transformation d'un tissu cellulaire différencié en un autre tissu cellulaire différencié. La dysplasie est une malformation ou déformation résultant d'une anomalie du développement d'un tissu cellulaire. Le terme néoplasie (littéralement: nouvelle croissance) désigne une formation nouvelle - le néoplasme - qui se développe par prolifération d'un nouveau type cellulaire différencié. La Figure 11 montre la simulation d'une recroissance néoplasique après 
traitement (évolutions, sous différents traitements, de la taille de la tumeur à gauche et simulation d'évolutions de coupes scanner $2 \mathrm{D}$ à droite, à partir d'un reliquat d'exérèse chirurgicale [35]). Le néoplasme se développe en sécrétant un facteur de croissance vasculaire attirant les vaisseaux artériels, processus dual du drainage veineux. La Figure 12 montre un drainage de forêt (en Suède) après une tempête et le drainage veineux du foie, réel et simulé $[36,37]$, processus qui, relevant du même type de graphe d'interactions, présentent la même phénoménologie.

\section{La « kairothérapie 》}

La prise en compte, dans le diagnostic et dans la thérapie des instants privilégiés du kairos permet d'envisager un suivi, calqué sur la temporalité de la maladie, pour vérifier si le traitement est efficace. Le meilleur exemple est la chronothérapie des cancers, au cours de laquelle le médecin essaie de trouver l'instant le plus efficace, celui auquel les cellules cancéreuses seront les plus sensibles à une drogue antimitotique ou à une radiothérapie, les cellules saines étant synchronisées de manière à y être les moins sensibles [38, 39]. Cette thérapie nécessite une modélisation prédictive précise de la dynamique cellulaire normale et pathologique [40].

\section{Production des phanères}

Un autre exemple d'application de la biologie des systèmes à la morphogenèse concerne les phanères ( $c$ 'est-à-dire les productions visibles de l'épiderme et du derme, comme les poils, ongles, plumes, etc.). Le système génique de

\begin{tabular}{|c|c|c|c|}
\hline Attracteurs & $\begin{array}{l}\text { Mode d'itération } \\
\text { séquentielle }\end{array}$ & $\begin{array}{c}\text { Mode d'itération } \\
\text { parallèle }\end{array}$ & $\begin{array}{l}\text { Tissus } \\
\text { floraux }\end{array}$ \\
\hline Point fixe 1 & 000100000000 & 000100000000 & Sépale \\
\hline Point fixe 2 & 000100010110 & 000100010110 & Pétale \\
\hline Point fixe 3 & 000000001000 & 000000001000 & Carpelle \\
\hline Point fixe 4 & 000000011110 & 000000011110 & Étamine \\
\hline Point fixe 5 & 110000000000 & 110000000000 & Inflorescence \\
\hline Point fixe 6 & 110000010110 & 110100010110 & Mutant \\
\hline Cycle 1 & - & $\begin{array}{l}000100010000 \\
000100000110\end{array}$ & - \\
\hline Cycle 2 & - & $\begin{array}{l}000000000000 \\
000100001000\end{array}$ & - \\
\hline Cycle 3 & - & $\begin{array}{l}000000010000 \\
000100001110\end{array}$ & - \\
\hline Cycle 4 & - & $\begin{array}{l}000000000110 \\
000100011000\end{array}$ & - \\
\hline Cycle 5 & - & $\begin{array}{l}000000010110 \\
000100011110\end{array}$ & - \\
\hline Cycle 6 & - & $\begin{array}{l}000000001110 \\
000000011000\end{array}$ & - \\
\hline Cycle 7 & - & $\begin{array}{l}000100000000 \\
110000010000\end{array}$ & - \\
\hline
\end{tabular}

Figure 8. Attracteurs du système décrit par le graphe d'interactions de la floraison d'Arabidopsis thaliana (où 0 remplace -1 ).

contrôle de la formation des phanères a pour graphe d'interaction celui de la Figure 13 [41]. II existe un seul attracteur point fixe physiologique dont le bassin représente l'essentiel (plus de $96 \%$ ) des conditions initiales possibles, et ce dans tous les modes de mise à jour et pour un rayon moyen de bassin maximal. Si l'on se place dans cet attracteur, on peut déterminer les morphogènes présents, dont l'expression est contrôlée par le réseau. Parmi ces morphogènes, deux dominent: I'un (BMP2, bone morphogenic protein 2) responsable de l'inhibition de la morphogenèse des cellules migrantes du derme formant le primordium, ancêtre embryologique $d u$ phanère considéré, l'autre (BMP7) étant un chimioattractant, activateur de cette migration [42]. La structure spatiale finale (Figure 13) en amas localisés de cellules migrantes, préfigurant la localisation des primordia, est issue de la simulation d'un modèle de réaction-diffusion [41], dans lequel la cause de la localisation des amas réside dans le fait que l'inhibiteur diffuse plus vite que l'activateur, phénomène très

Figure 9. La base IMGT. Interrogation de la base IMGT: exemple d'un réarrangement de type $V 5 / J 53$. 


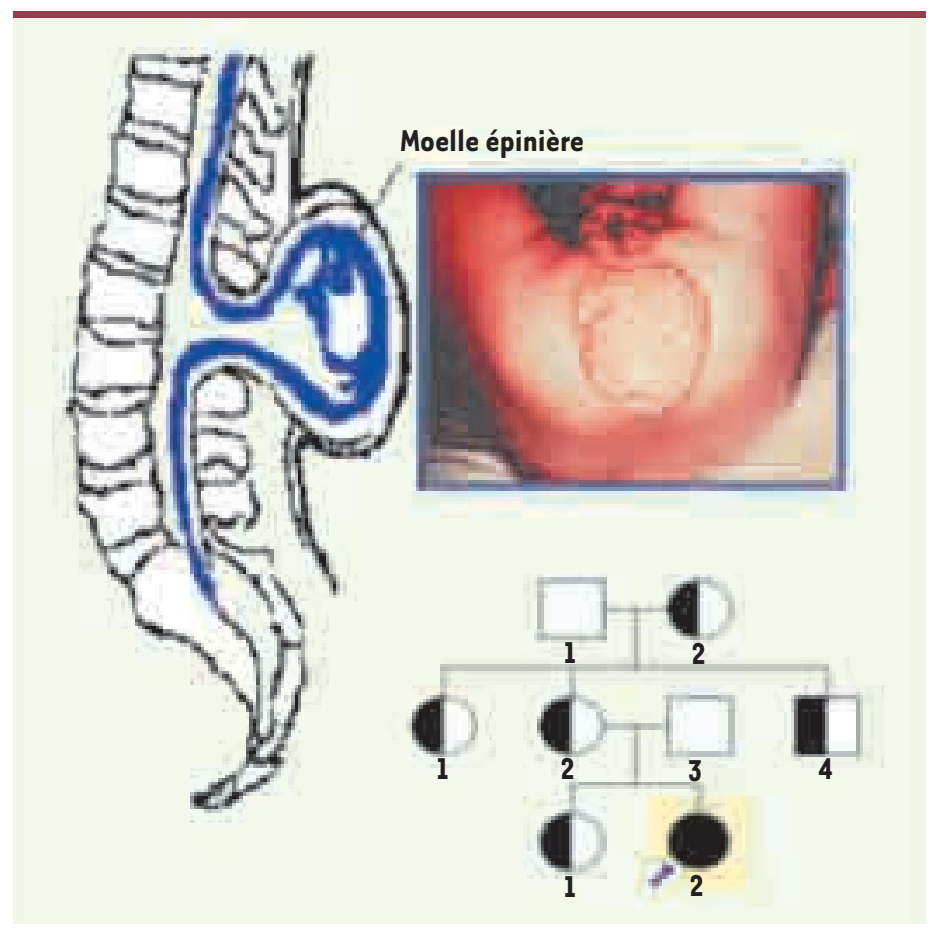

Figure 10. Applications de la biologie des systèmes en génétique médicale. Exemple de dysgenèse, la spina bifida, avec l'arbre généalogique du patient (noir), les porteurs sains des gènes pathologiques (noir et blanc) et les non-porteurs (blanc).

est transformé en ATP par une autre enzyme fixée sur la membrane interne de la mitochondrie, l'ATPase, et sort vers le cytosol grâce à la translocase. Les sites actifs de ces enzymes sont localisés au hasard sur la membrane interne et développent des interactions de type activation latérale (Figure 14) : en effet, à distance moyenne, ils s'attirent grâce à une force électromagnétique provoquée par des champs électriques locaux de signes différents (directement liés aux concentrations locales des adénylates, I'ATP ayant plus de charges négatives que l'ADP) et à une force de type van der Waals, tandis qu'à courte distance, ils se repoussent du fait du mouvement brownien thermique. La minimisation du libre parcours moyen de I'ADP et de l'ATP provoque donc, si la membrane interne est libre (sous des contraintes de courbure fixées par la concentration locale de cardioli-

général en morphogenèse et en perception (par exemple en vision ou en audition), désigné sous le nom générique d'inhibition latérale.

\section{Applications de la biologie des systèmes en biochimie médicale}

L'énergétique mitochondriale est optimale si le libre parcours moyen des adénylates (ADP et ATP) est minimal [11]: en effet, l'ADP entre dans la mitochondrie à l'aide d'une enzyme transmembranaire, la translocase, pine, substance fixant la plasticité de la structure phospholipidique), une structuration en crêtes (Figure 14 en bas à droite). Le nombre de crêtes est directement lié à l'efficacité de la respiration cellulaire et I'on peut simuler le système complexe correspondant aux adénylates et aux enzymes qui les transportent et les transforment [11] de manière à mieux comprendre les mécanismes produisant des mitochondries pathologiques (avec par exemple une seule crête enroulée en «pelure d'oignon » due à une mutation de l'ATPase) ou ceux causant la réaction mitonchondriale à une anoxie de type post-infarctus, c'est-à-dire l'augmentation du nombre des crêtes (facilitée par un traitement à base de cardiolipine) ou la division mitochondriale.

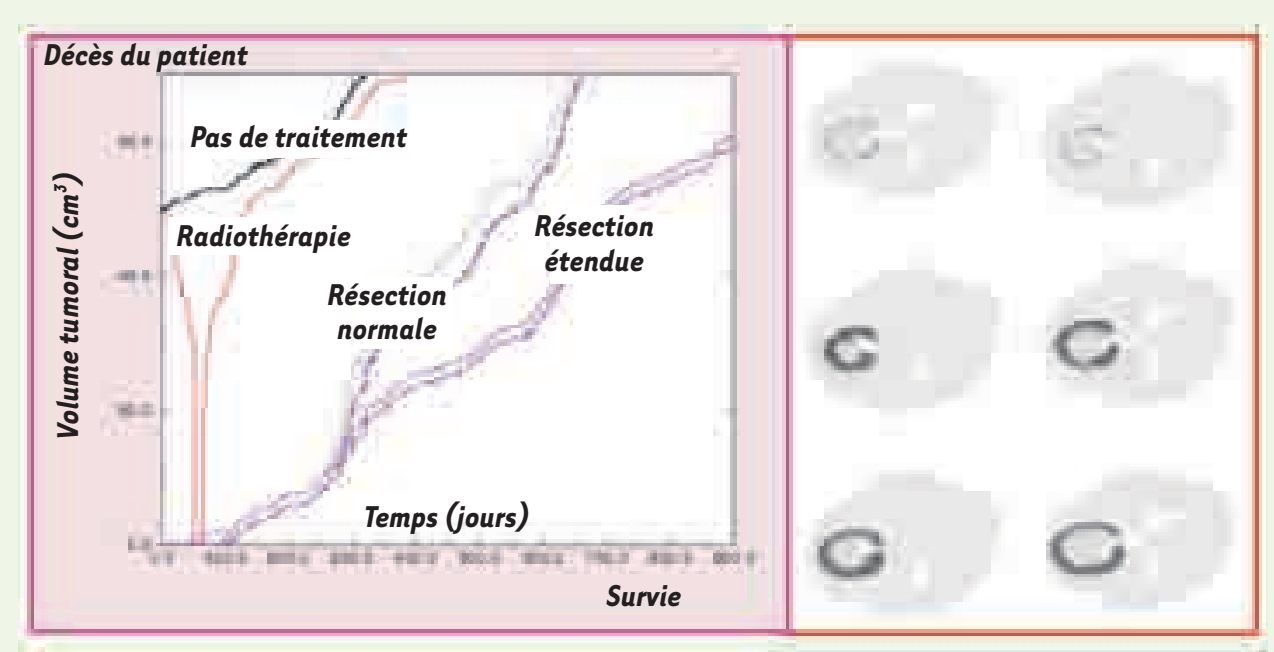

Figure 11. Simulation d'une croissance néoplasique. La croissance néoplasique (à droite) et sa prédiction thérapeutique (à gauche).
Applications de la biologie des systèmes au savoir médical : télémédecine et multi-expertise

Le savoir médical [43] est, nous l'avons vu, distribué dans des bases de données de plusieurs types: savoir statistique des bases de données patients [44, 45], savoir encyclopédique de l'université médicale virtuelle $[46,47]$ et savoir expert des spécialistes [48]. Les réseaux de copublication 


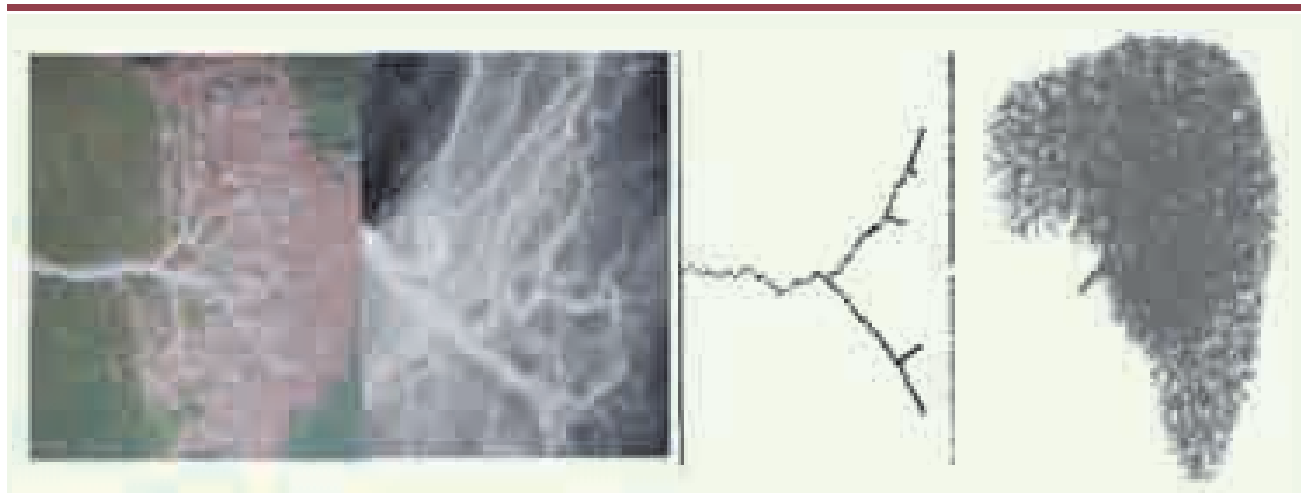

Figure 12. Autres exemples de simulation de croissance. Croissance de chemins forestiers (gauche), du hile veineux cave hépatique (centre gauche), d'une veine simulée de drainage (centre droit) et du hile veineux cave simulé (droite).

$[49,50]$ permettent, dans ces gisements du savoir médical, de cerner des zones de connaissance médicale partagée et de coexpertise (Figure 15), éléments indispensables de la médecine plurielle. Prenons l'exemple d'une maladie commune en Afrique: le paludisme. Un médecin généraliste français, à la recherche d'une expertise de première intention, pour orienter le diagnostic et chercher ensuite sur place le médecin spécialiste (cytologiste ou anatomopathologiste) qui l'affirmera, pourra par exemple choisir le chemin suivant sur Internet:

- Google/mots-clés : paludisme, patient européen, séjour récent Cameroun.

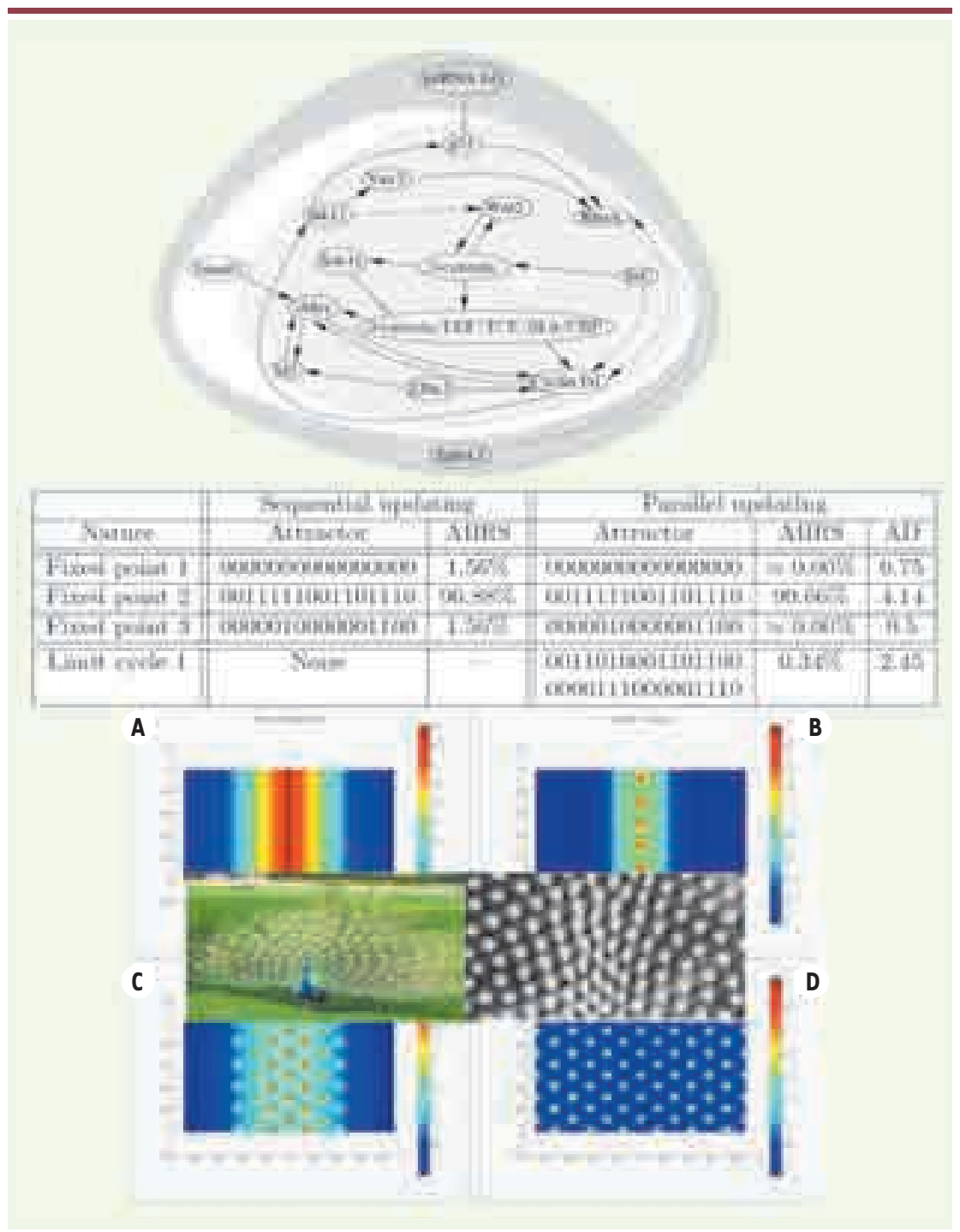

- Google/mots-clés : paludisme, médecine tropicale, Grenoble.

S’il désire un spécialiste d'emblée, pour un conseil thérapeutique en vue d'une thérapie présomptive au retour d'un séjour africain, le chemin sera:

- Google/mots-clés: malaria network, tropical medicine, presumptive therapy.

- Kartoo/recherche : nom des spécialistes suggérés par Google [49].

Le médecin généticien humain, consulté pour un conseil génétique dans une

Figure 13. Applications de la biologie des systèmes en morphogenèse. Le développement embryonnaire du réseau plumaire chez l'oiseau permet par exemple le placement précis des plumes pour l'érection de la roue chez le paon (en bas). Graphe d'interaction du réseau contrôlant la synthèse des morphogènes (en haut). Attracteurs du système et pourcentage des états dans leurs bassins (ABRS), ainsi que rayon moyen des bassins $(A D$, en distance de Hamming moyenne à l'attracteur) (au centre). Simulation de la morphogenèse (en bas): $A$. cellules primordiales épidermiques de l'axe central dorsal; B. taches de morphogènes attirant les cellules migrantes du derme; $\boldsymbol{C}$. migration cellulaire sous l'effet d'un gradient latéral d'inhibiteur; $\boldsymbol{D}$. formation plumaire embryonnaire hexagonale finale, à comparer à la formation réelle (au dessus). 


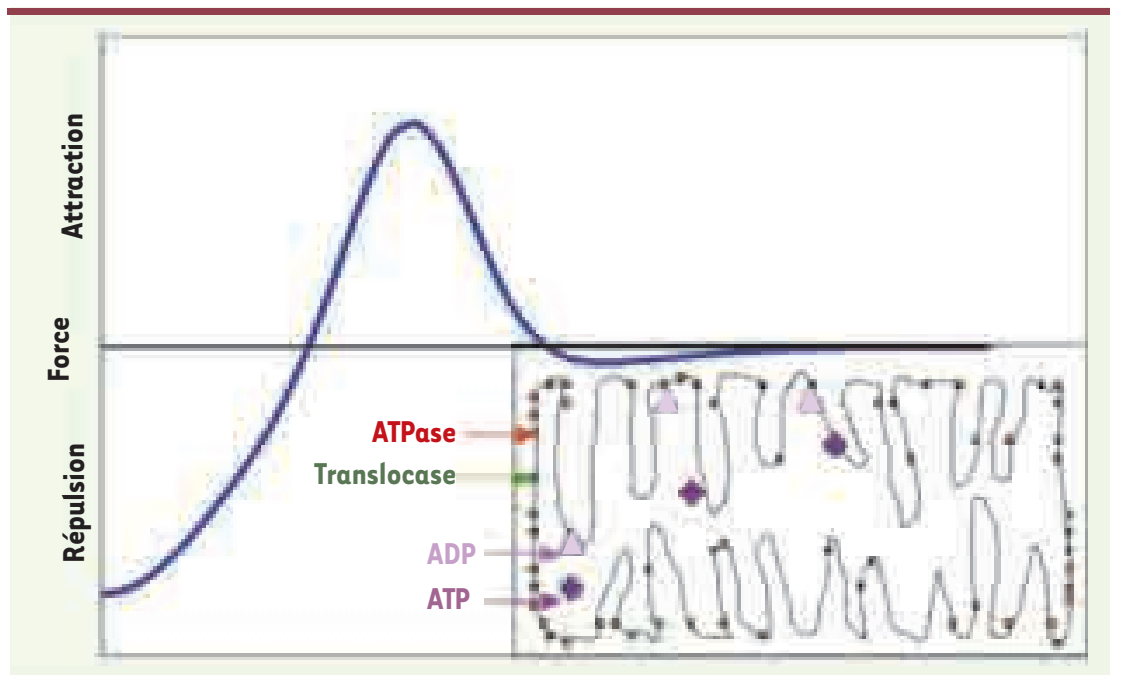

Figure 14. Applications de la biologie des systèmes en morphogenèse. Représentation du champ de forces de type activation latérale mis en œuvre dans la formation des crêtes mitochondriales (en bas à droite) rapprochant les sites enzymatiques ATPases (en rouge) et translocases (en vert), de manière à optimiser le libre parcours moyen des adénylates (ADP et ATP). cas de paludisme, et ce avec une très bonne fiabilité et une grande précision.

\section{Vers un dossier « clinomique »}

Nous avons vu plus haut l'intrication des niveaux auxquels sont obtenues les données nécessaires au diagnostic et au suivi thérapeutique. II est clair que la médecine moderne exige de stocker, puis de fournir rapidement (par exemple en urgence), les informations relatives à un patient, de manière à accélérer les procédures diagnostiques. Les bases de données encyclopédiques médicales ont environ 30 ans: la première française fut la base ADM, aide au diagnostic médical du $\operatorname{Pr}$ M. Lenoir, accessible dès l'origine par Minitel. Les plus interrogées se trouvent actuellement à la National library of medicine et à l'UMVF (Université médicale maladie orpheline familiale d'origine chromosomique, dont on a observé le caryotype pathologique montrant une translocation impliquant les bras longs des chromosomes 11 et 22, suivra un chemin différent pour conforter son hypothèse diagnostique par une expertise de seconde main, en utilisant une base de connaissances cytogénétique, Human chromosome forum, dite HCForum ${ }^{\circledR}$ et des moteurs publics de recherche de copublication, PubNet et Kartoo :

- HCForum ${ }^{\circledR} /$ mots-clés : translocation $11 / 22$, short report (Figure 16) [50].

- PubNet/recherche: réseau de copublication d'un auteur cité par HCForum ${ }^{\circledR}$ (Figure 15) [51].

- Kartoo/recherche : réseau sémantique de la maladie orpheline indiquée par HCForum ${ }^{\circledR}$, à savoir le syndrome de retard mental/polymalformation (Figure 17) [49].

\section{Applications de la biologie des systèmes à l'épidémiologie}

Une perspective immédiate d'application réside dans la surveillance et le conseil à distance, pour la prévention de maladies épidémiques, endémiques ou familiales sporadiques $[8,39]$ : la Figure 18 présente un exemple de suivi de cas de paludisme dans un village du Mali (Bancoumana [52]). Dans cet exemple, les images satellite SPOT permettent la détection (à $20 \mathrm{~cm}$ près) de zones humides avec eaux stagnantes (essentiellement mares et marigots) au voisinage du village, donc la prédiction des lieux de ponte de moustiques, et enfin le calcul de l'occurrence spatio-temporelle de nouveaux virtuelle francophone) $[46,47]$, qui permettent des requêtes dans le savoir académique enseigné dans la formation médicale initiale. Dans les bases de données statistiques classiques centrées sur les patients, on a d'abord résolu le problème du stockage en structurant l'information sur le mode réseau et en fournissant des repères moyens permettant de regrouper les patients par GHD (groupes homogènes de diagnostic) [53]. Ce fut le cas à Grenoble de la base DOSTAM (dossier statistique de l'activité médicale), construite en 1967 selon le modèle $Z$ de J.R. Abrial [54]. À l'intérieur d'un groupe homogène, diverses variables discriminantes permettent d'affiner les classifications

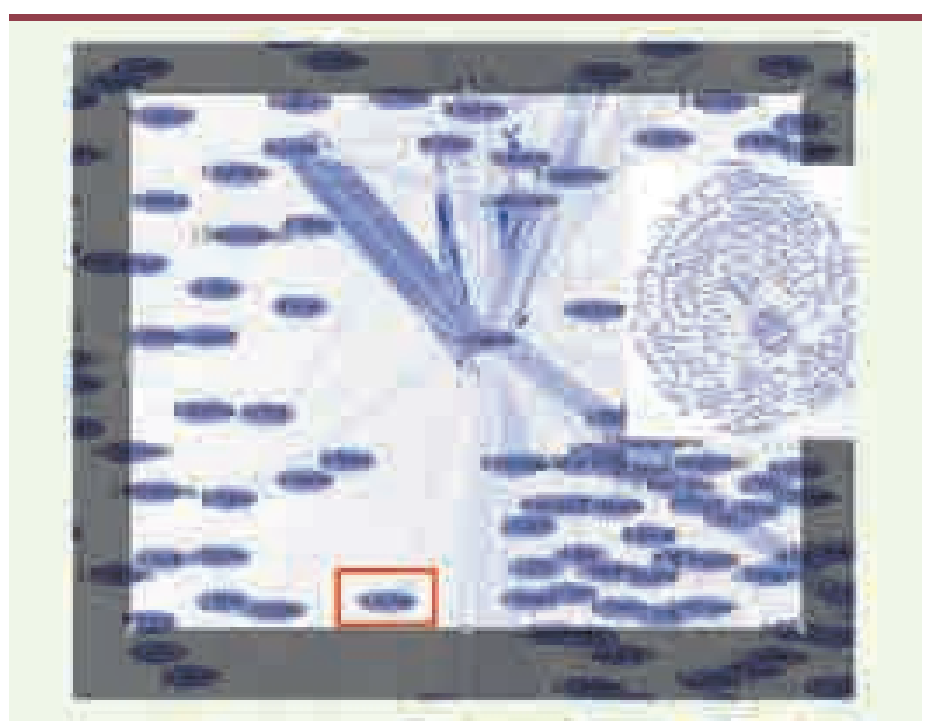

Figure 15. Réseaux de copublication. Exemple d'un réseau centré sur J. Lejeune ; réseau entier (en médaillon) et lien avec l'auteur, A. Aurias, fourni par HCForum ${ }^{\circledR}$. 


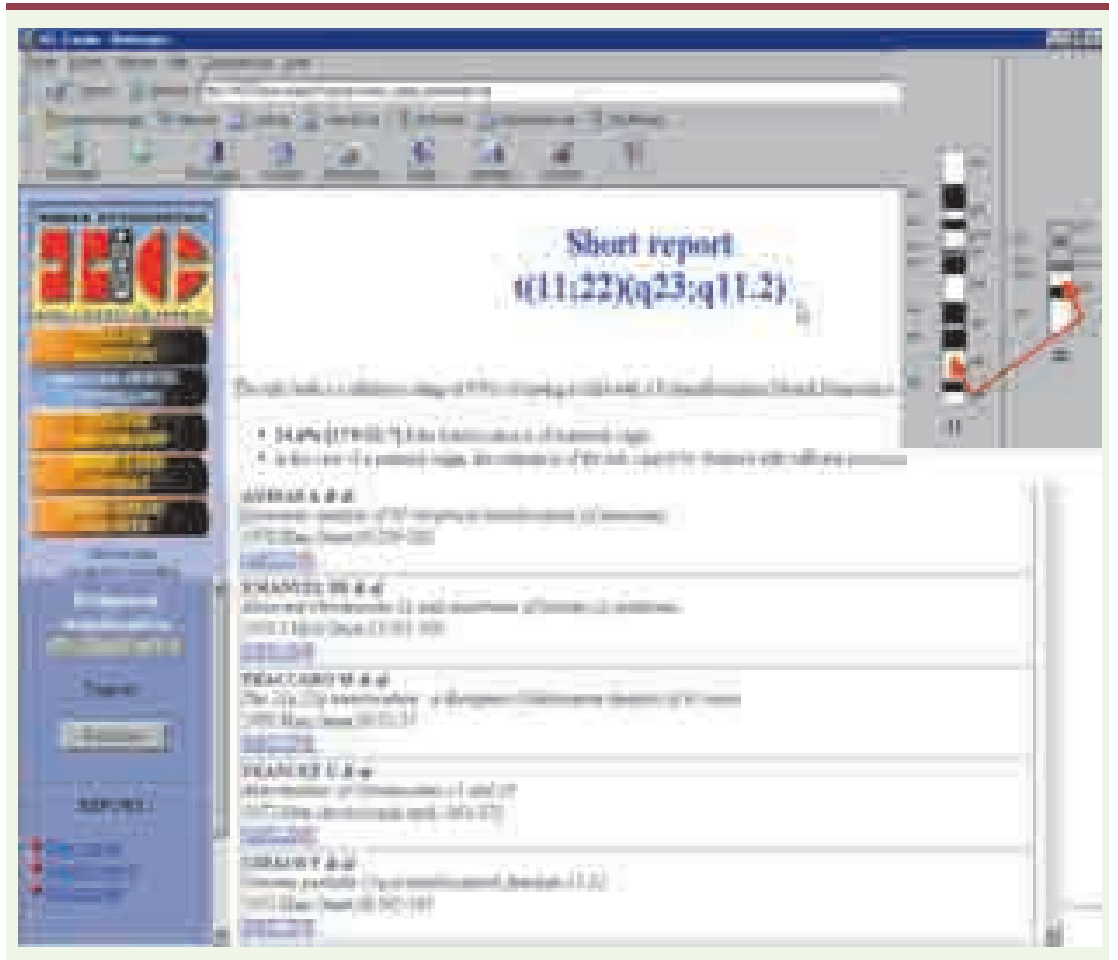

Figure 16. Recherche biblographique sur HCForum ${ }^{\circledR}$. La recherche est effectuée à partir de l'observation, sur un caryotype, d'une translocation entre les chromosomes 11 et 22. on calcule par exemple les distributions de probabilité des positions spatiales et des courbures en leurs singularités (Figure 20). La mise en correspondance statistique se fait ensuite en utilisant des distances classiques, euclidiennes ou entropiques, comme dans le système HELP de Salt Lake City, créé en 1967 par H.R. Warner [55], dont la description en 1975, prônant la décision statistique, fut éclipsée par l'article princeps sur le premier système expert médical MYCIN écrit par E.H. Shortliffe et B.G. Buchanan [56]. Depuis, les bases statistiques ont réaffirmé leur primauté, mais l'expertise reste présente dans des systèmes où les 3 formes de savoir médical sont liées, comme dans HC Forum ${ }^{\circledR}$, ce qui permet le conseil génétique à distance [51].

Ces bases nécessitent des requêtes hybrides, à la fois numériques et symboliques, appelant des algorithmes qui permettent le matching entre le patient et la référence, à travers un modèle de régulation par exemple, et autorisent la description déclarative clinique. Ces requêtes peu-

nosologiques de référence comme la CIM 10 (classification internationale des maladies). Un exemple de telles variables est la durée de la maladie, souvent confondue avec la durée du séjour hospitalier. Celle-ci a souvent une distribution multi-modale $[44,45]$, ce qui permet de démembrer les diagnostics nouveaux ou rares, de manière à affiner la nosologie (Figure 19). Le savoir statistique est également présent à travers les atlas, qui sont des bases de données d'organes dont

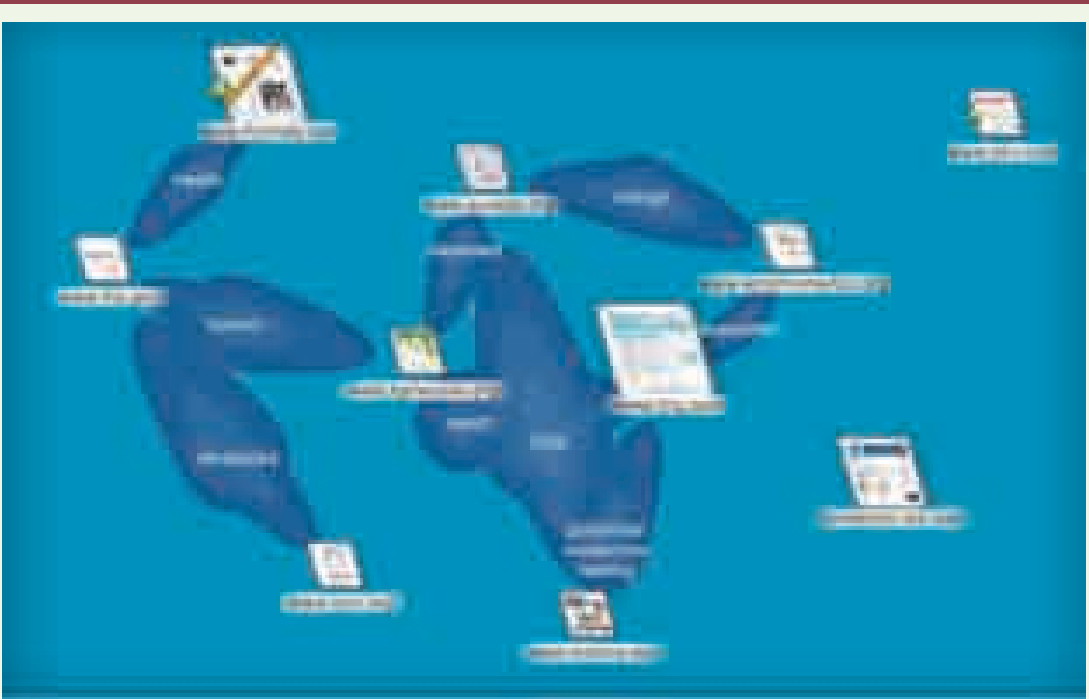

vent partir d'un dossier résumé de type DOSTAM (30 kb), puis utiliser un dossier clinique $(300 \mathrm{~kb})$, ainsi que des images radiologiques (3 Mb) ou même actuellement des données d'interactions géniques et/ ou protéiques ( $3 \mathrm{~Gb}$ ), constituant ainsi un nouveau dossier dit «clinomique », centré sur le patient. La hiérarchisation et l'optimisation de requêtes «clinomiques » font partie des problèmes les plus difficiles de l'informatique actuelle et seule la théorie des systèmes complexes permet de ramener leur durée à des temps d'exécution polynomiaux. La théorie des systèmes complexes, fondement mathématique de la biologie des systèmes, permet:

- de placer un patient dans un réseau spécifique de groupes homogènes d'individus en interaction, par suite de rencontres (dans le cas des maladies transmissibles) ou de pedigrees consanguins (dans le cas des maladies familiales). La modélisation de ce réseau est actuellement possible grâce à des algorithmes appelés individus-centrés ou multi-agents;

- de mettre à disposition du clinicien un réseau général de référence, constitué

Figure 17. Exemple d'un réseau de maladie orpheline. Le syndrome de retard mental/ polymalformation. 
des bases de patients des diverses institutions de soins (de proximité, de recours ou de référence universitaire), reliant entre eux des groupes homogènes de malades.

\section{Conclusion}

Dans le triangle hippocratique classique $[57,58]$, l'art médical se décline en trois acteurs, les trois $M$ : la maladie «M la maudite», le malade «M le misérable» et le médecin «M le médiateur ». Le médecin est le desservant de l'art et il faut que le malade aide le médecin à combattre la maladie qui est leur ennemie commune [59]. Le pentagone médical moderne comporte cinq $M$ : le modèle «M le mathématique », le malade, la maladie, le médecin et le médicament «M le miraculeux ». Chaque nouvel ou ancien acteur a sa place dans la tragédie médicale. L'apport du modèle est devenu incontournable pour comprendre l'étiologie de la maladie, orienter un diagnostic et trouver un traitement approprié au patient. L'ordinateur permet ainsi de visualiser jusqu'au résultat futur de la thérapie, par exemple en chirurgie esthétique. Le malade nouveau est devenu savant et les associations de patients sont les principaux artisans du financement de la recherche médicale. La maladie s'est adaptée, phénomène dont sont témoins les agents infectieux qui développent des stratégies complexes pour échapper au médicament. Dans ce contexte, le médecin a évolué, conscient de sa faiblesse s'il est seul en face de la maladie. Les hippocratiques anciens ou récents observaient déjà l'humilité : «ils considéraient qu'il était bon que le médecin avoue ou au moins reconnaisse, le cas échéant, son incompétence et sollicite l'aide d'autres médecins » [60]. Le médecin se comporte de plus en plus comme un «passeur»,

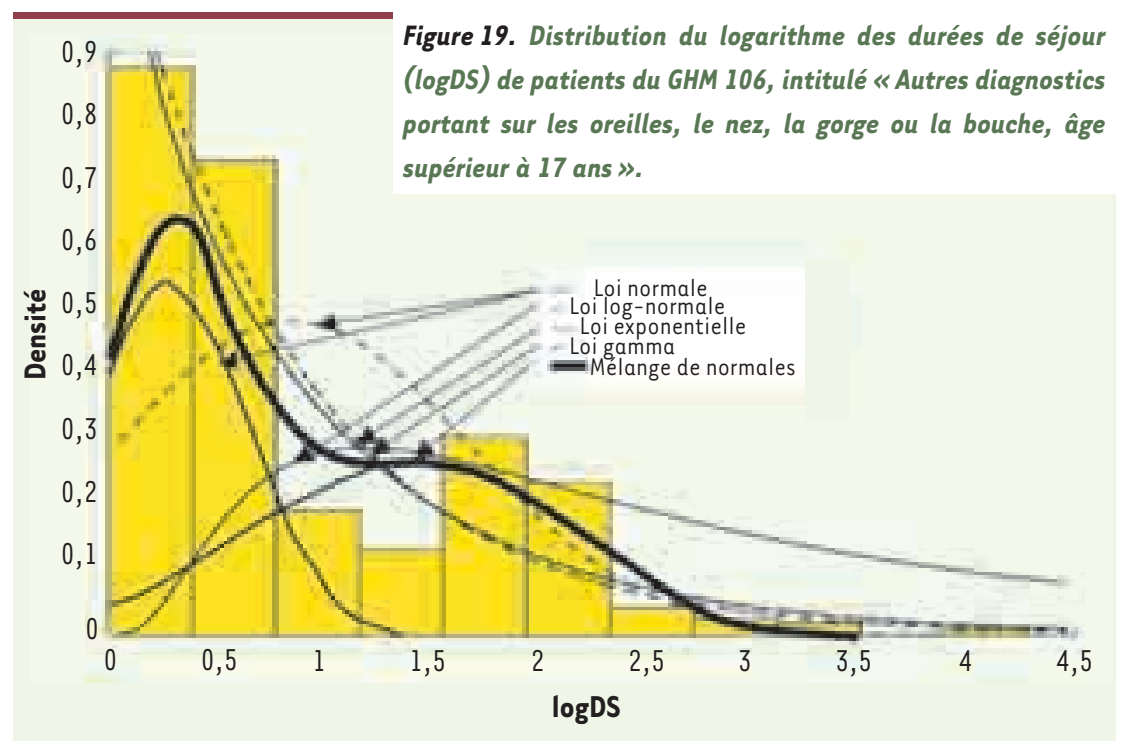

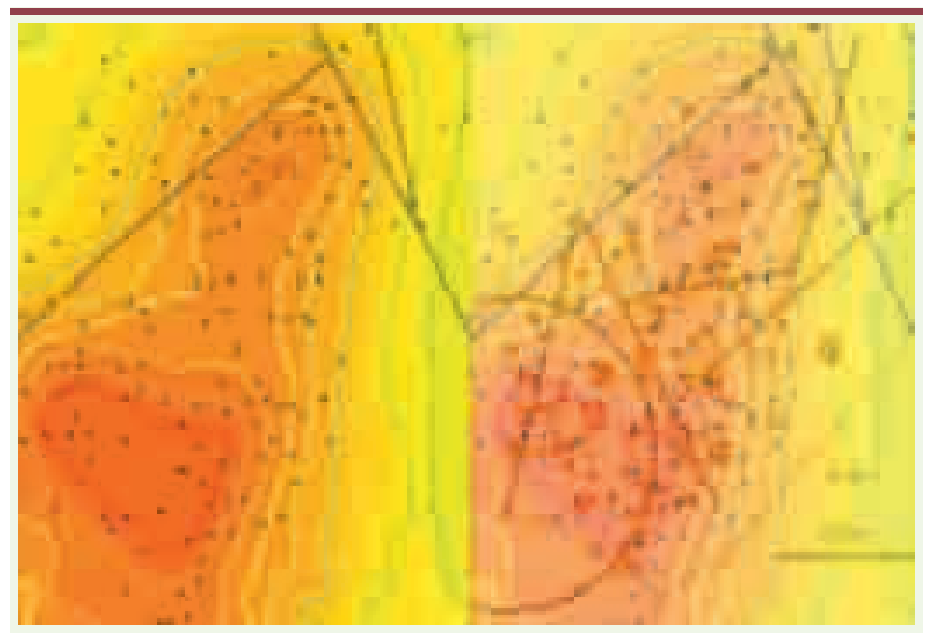

Figure 18. Utilisation d'un système d'information géographique en surveillance épidémique. Une équation de diffusion-réaction permet de prévoir un gradient de malades sur des groupes de cases (à gauche) correspondant bien à la prévalence observée (à droite). Les zones de maximum (resp. minimum) de prévalence sont en rouge (resp. vert).

vers une pluri-expertise rendue rapide et fiable par l'usage de l'internet. Enfin, le médicament constitue désormais une entité multiple, aux frontières floues entre micro-nano-dispositifs et molécules d'intérêt diagnostique et/ou thérapeutique: un bon exemple de cette confusion est l'administration d'une drogue par un container guidé par une micro-caméra qui délivre dans le tube digestif un isotope lié à un vecteur liposomique et à une molécule immunocompétente, capables de franchir la paroi intestinale et d'aller électivement se fixer sur les protéines pathologiques des parois d'une cellule tumorale de carcinose péritonéale, afin de repérer et tuer les cellules cancéreuses des nodules tumoraux secondaires à une tumeur primitive, par exemple du col utérin. Ce nouveau médicament complexe constitue un servant des quatre autres acteurs du pentagone médical moderne, comme le faisait la drogue de la pharmacopée grecque dans le triangle hippocratique ancien, constituant ainsi la base rationnelle de la médecine contemporaine. $\diamond$

\section{SUMMARY}

Medical applications of systems biology We illustrate in this review some applications of systems biology in the medical and biological areas. After a brief summary of time scales experienced by medical observations and of the general scheme of dynamic systems, we describe how some techniques underlying the complex systems theory can be applied to model medical issues in immunology, medical genetics, developmental mor- 


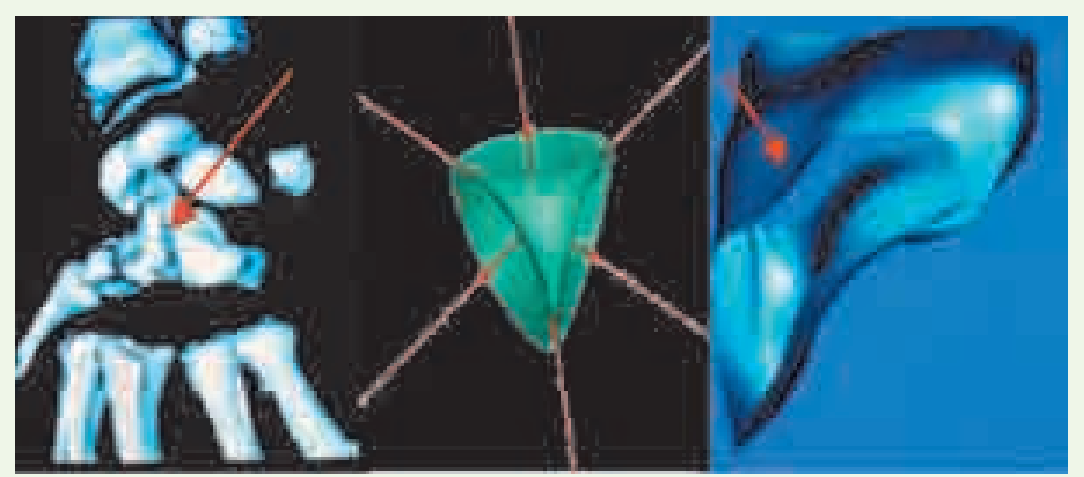

Figure 20. Points singuliers de Withney d'une surface, présents sur les os du poignet (grand os à gauche), sur la surface de Steiner (au centre) et sur le noyau gris central VIM (ventralis intermedius nucleus), permettant la mise en correspondance dans les atlas statistiques.

phogenesis, biochemistry, epidemiology, telemedecine and multiple platforms of expertises. In concluding, we will discuss the issue of "clinomics" coupling clinical and -omics data in a unique patientspecific file. $\diamond$

\section{RéFÉRENCES}

1. Galton F. Psychometric experiments. Brain $1879 ; 2: 149-62$

2. Priftis K, Zorzi M, Meneghello F, et al. Explicit versus implicit processing of representational space in neglect: dissociations in accessing the mental number line. J Cognitive Neurosci 2006 ; $18: 680-8$.

3. De Beni R, Pazzaglia F, Gardini S. The generation and maintenance of visual mental images: evidence from image type and aging. Brain Cognition $2007 ; 63: 271-8$.

4. Klee P. La Pensée créatrice. Paris: Dessain et Tolra, 1973.

5. Poincaré H. CFuvres, 11 vol. Paris : Gauthier-Villars, 1913-1965.

6. Kauffman S. Origins of order: self-organization and selection in evolution. Oxford: 0xford University Press, 1993.

7. Michon F, Forest L, Collomb E, et al. BMP-2 and BMP-7 play antagonistic roles in feather induction. Development $2008 ; 135: 2797-805$.

8. Demongeot J, Elena A, Sené S. Robustness in neural and genetic networks. Acta Biotheor 2008 ; $56: 27-49$.

9. Demongeot J, Moreira A. A circular RNA at the origin of life. J Theor Biol $2007 ; 249: 314-24$.

10. Demongeot J, Glade N, Moreira A. Evolution and RNA relics. A Systems Biology view. Acta Biotheor $2008 ; 56: 5-25$.

11. Demongeot J, Glade N, Hansen 0, Moreira A. An open issue: the inner mitochondrial membrane (IMM) as a free boundary problem. Biochimie $2007 ; 89$ : 1049-57.

12. Cinquin 0 , Demongeot J. Positive and negative feedback: striking a balance between necessary antagonists. J Theor Biol $2002 ; 216: 229-41$.

13. Delbrück M. Unités biologiques douées de continuité génétique. In : Colloques internationaux du Centre national de la recherche scientifique. Paris : Éditions du CNRS, 1949 : 33-4.

14. Meyer $\varepsilon$, Beisson J. Épigénétique : la paramécie comme modèle d'étude. Med Sci (Paris) 2005 ; 21 377-83.

15. Cinquin 0, Demongeot J. Positive and negative feedback: mending the ways of sloppy systems. Comptes Rendus Biologies 2002 ; 325 : 1085-95.

16. Kaufman M, Soulé $C$, Thomas R. A new necessary condition on interaction graphs for multistationarity. J Theor Biol 2007 ; $248: 675-85$.

17. Aracena J, Demongeot J, Goles $\varepsilon$. Fixed points and maximal independent sets on AND-OR networks. Discrete Appl Math 2004 ; $138: 277-88$.

18. Aracena J, Demongeot J, Goles $\varepsilon$. Mathematical modelling in genetic networks. IEદદ T Neural Networks $2004 ; 15: 77-83$.

19. Goles $\varepsilon$, Olivos J. Comportement périodique des fonctions à seuils binaires et applications. Discrete Appl Math 1981; 3 : 93-105.

20. Aracena J, Ben Lamine S, Mermet MA, et al. Mathematical modelling in genetic networks: relationships between the genetic expression and both chromosomic breakage and positive circuits. IEદE T Syst Man Cy 2003 $33: 825-34$.

21. Goles $\varepsilon$, Salinas L. Comparison between parallel and serial dynamics of Boolean Networks. Theor Comput Sci 2008 ; $396: 247-53$.

22. Salinas L. Discrete models: structure and dynamics. Chili : PhD Thesis, Universidad de Chile, 2008.

23. Kauffman S, Peterson C, Samuelsson B, Troein C. Random Boolean network models and the yeast transcriptional network. Proc Natl Acad Sci USA 2003; 100 : 14796-9.
24. Kauffman S, Peterson C, Samuelsson B, Troein C. Genetic networks with canalyzing Boolean rules are always stable. Proc Natl Acad Sci USA 2004 ; $101: 17102-7$

25. Aldana-Gonzalez M, Coppersmith S, Kadanoff LP. Boolean dynamics with random couplings. In : Kaplan $\varepsilon$, Marsden JE, Sreenivasan KR, eds. Perspectives and problems in nonlinear science. Applied mathematical sciences series. Berlin : Springer, 2003 : 23-89.

26. Karlsson F, Hörnquist M. Order or chaos in Boolean gene networks depends on the mean fraction of canalizing functions. Physica A Stat Mechanics Applications 2007 ; 384 : 747-57.

27. Demongeot J, Jezequel C, Sené S. Asymptotic behavior and phase transition in regulatory networks. I. Theoretical results. Neural Networks 2008; 21 : 962-70.

28. Demongeot J, Sené S. Asymptotic behavior and phase transition in regulatory networks. II. Simulations. Neural Networks $2008 ; 21$ : $971-9$.

29. Mendoza L, Alvarez-Buylla $\varepsilon$ R. Dynamics of the genetic reglatory network for Arabidopsis thaliana flower morphogenesis. J Theor Biol 1998 ; 193 : 307-19.

30. Sené $S$. Influence des conditions de bord dans les réseaux d'automates booléens à seuil et application à la biologie. Lyon : Thèse, Université J. Fourier et ENS Lyon, 2008.

31. Baum TP, Pasqual N, Hierle V, et al. IMGT/Genelnfo: new gamma and delta chains for database V(D)J recombination. BMC Bioinformatics 2006 ; $7: 224-8$.

32. Baum TP, Pasqual N, Thuderoz F, et al. IMGT/Genelnfo: enhancing V(D)J recombination database accessibility. Nucleic Acids Res $2004 ; 32$ : 51-4.

33. Bidot C, Gruy F, Haudin CS, et al. Mathematical modelling of T-cell activation kinetic. J Comput Biol 2008 ; 15 : 105-28.

34. Cohen 0, Mermet MA, Demongeot J. HC Forum: a web site based on an international human cytogenetic database. Nucleic Acids Res 2001; 29 : $305-7$.

35. Woodward D. E, Cook J, Tracqui P, et al. A mathematical model of glioma growth: the effect of extent of surgical resection. Cell Proliferation 1996; $29: 269-88$.

36. Kergosien YL. Adaptive ramification: comparing models for biological, economical, and conceptual organization. Acta Biotheor $1990 ; 38$ : 243-55.

37. Demongeot J, Bezy-Wendling J, Mattes J, et al. Multiscale modeling and imaging: the challenges of biocomplexity. Proc IEદE Society 2003; 91 : 1723-37.

38. Bernard-Weil $\varepsilon$. Is it possible to equilibrate the different "levels" of an imbalanced biological system by acting upon one of them only? Example of the agonistic antagonistic networks. Acta Biotheor 1991; 39:271-85.

39. Lévy F. Circadian chronotherapy for human cancers. Lancet Oncol $2001 ; 2$ : 307-15.

40. Cinquin 0, Demongeot J. High-dimensional switches and the modeling of cellular differentiation.J Theor Biol 2005 ; 233 : 391-411.

41. Ben Amor H, Cadau S, Demongeot J, Sené S. Regulatory networks analysis: robustness in artificial and real biological networks. In : IEદE CISIS'09 and IIBM'09. Piscataway : Iદદદ Proceedings, 2009 : 224-9.

42. Forest L, Michon F, Cadau S, et al. What is the biological basis of pattern formation of skin lesions? Viewpoint 4. Skin patterns belong to three main types, determined at three steps of development. Exp Dermatol 2006 ; $12: 559-64$.

43. Demongeot J. Le traitement de la connaissance médicale et son apprentissage. Informatique et Santé 1994 ; 18 : 27-31.

44. Delhumeau C. Contribution à la modélisation des durées de séjour du CHU de Grenoble. Grenoble : Thèse UJF, 2002. 
45. Langlois C. Modélisation des durées de séjour. Grenoble: Rapport interne du CHU de Grenoble, 2003.

46. Le Beux P, Benhamou AC, Beuscart R, et al. A common platform for French virtual medical universities. In : Challenges primary care-oriented health system. Abingdon : Londrina, The Network Education Health : 2001 : 101-3.

47. URL: www.umvf.org.

48. Cohen 0 , Mermet MA, Demongeot J. HC Forum ${ }^{\circledR}$ or when cytogenetics meets telemedicine. Annales de génétique $2001 ; 44: 9$.

49. URL: http://pubnet.gersteinlab.org/.

50. URL: http://www.kartoo.com/fr_index.htm.

51. Cohen 0, Mermet MA, Demongeot J. HC Forum ${ }^{\circledR}$ : toward a tele-expertise plat-form in medical genetics. Lecture Notes Med Inf 2002 ; 13 : 97-104.

52. Gaudart J, Giorgi R, Poudiougou B, et al. Spatial cluster detection: principle and application of different general methods. Rev Epidémiol Santé Publ $2007 ; 55: 297-306$.

53. Valois J, Abrial JR, Cohen S. Description d'un système de gestion en temps réel du dossier médical. Grenoble Médico-Chirurgical 1970 ; 8 : 301-14.
54. Fetter RB, Freeman JL. Diagnosis related groups: product line management within hospitals. Acad Manag Rev 1986; $11: 41-54$.

55. Pryor TA, Morgan JD, Clark S J, Miller WA, Warner HR. Help. A Computer assisted system for medical decision making. Computer $1975 ; 8: 34-8$.

56. Shortliffe $\varepsilon H$, Buchanan BG. A model of inexact reasoning in medicine. Mathematic Biosci 1975; $23: 351-79$.

57. A. Klein. On ne peut apprendre rapidement la médecine. Le Portique, e-portique 2007 ; 4 : URL http://leportique.revues.org/document1011.html.

58. Hippocrate. Guvres complètes II. Épidémies I, 5. Traduction $\varepsilon$. Littré. Paris : J.B. Baillère, 1846.

59. Gourevitch D. Le triangle hippocratique dans le monde gréco-romain. Le malade, sa maladie et son médecin. Bulletin des Écoles Françaises d'Athènes et de Rome $1984 ; 251$.

60. Sow Diouf M. Étude de déontologie médicale hippocratique. Ethiopiques $2004 ; 72: 3$.

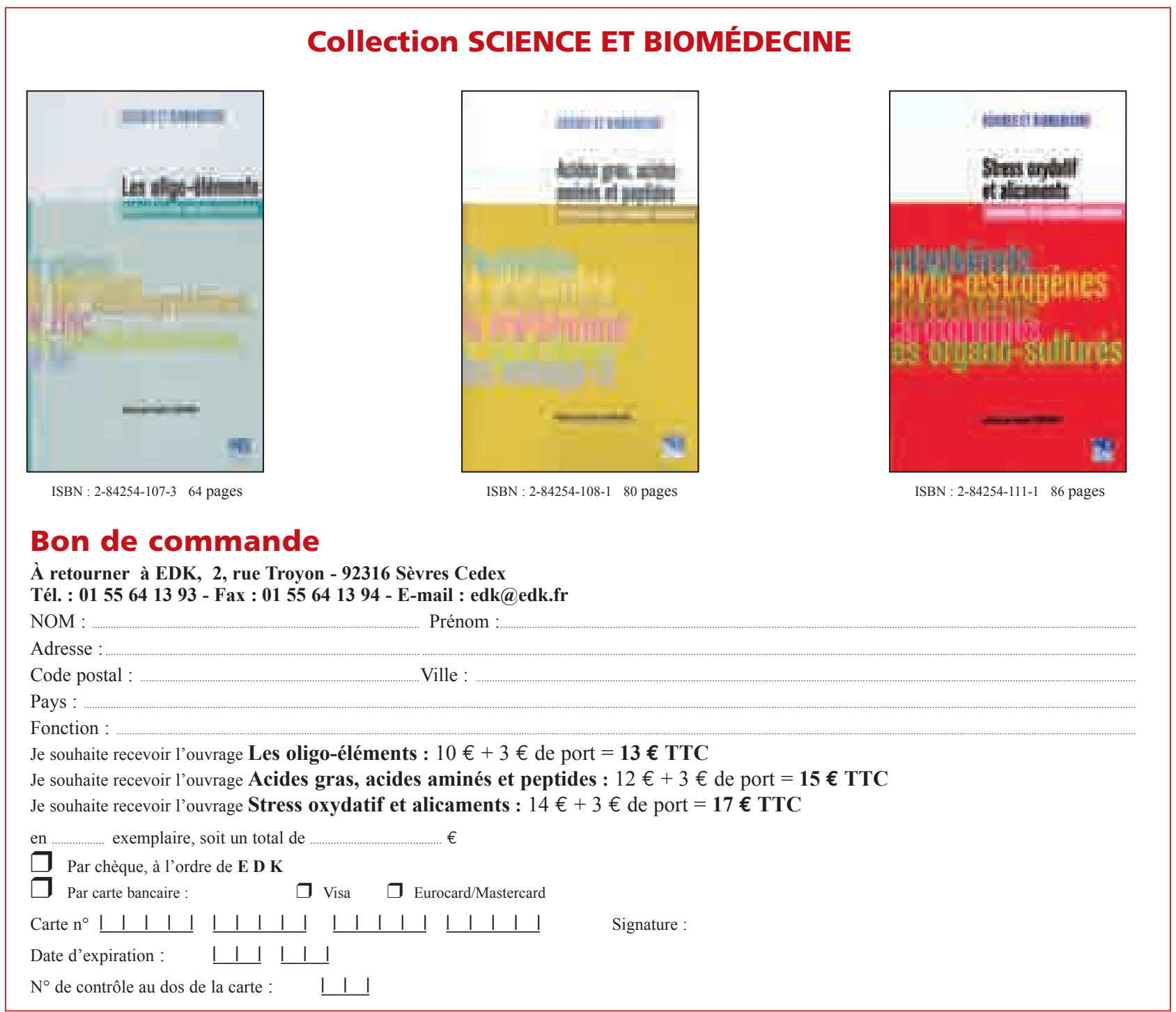

